\title{
Splenic Abscess Complicating Scrub Typhus Induced Splenic Infarct - A Rare Case Report
}

\author{
Akhilesh Annadatha ${ }^{1}$, Sourya Acharya², Amol Andhale³, Sree Karthik Pratapa ${ }^{4}$
}

1Department of Medicine, Datta Meghe Institute of Medical Sciences (Deemed to Be University) Sawangi Meghe, Wardha, Maharashtra, India. ${ }^{2}$ Department of Medicine, Datta Meghe Institute of Medical Sciences (Deemed to Be University) Sawangi Meghe, Wardha, Maharashtra, India. ${ }^{3}$ Department of Medicine, Datta Meghe Institute of Medical Sciences (Deemed to Be University) Sawangi Meghe, Wardha, Maharashtra, India. ${ }^{4}$ Department of Medicine, Datta Meghe Institute of Medical Sciences (Deemed to Be University) Sawangi Meghe, Wardha, Maharashtra, India.

\section{INTRODUCTION}

Any condition leading to the interruption of blood flow to the spleen, such as blockage in the splenic artery due to a plaque or a clot in one of its branches leads to Splenic Infarction. It could also be due to infection, trauma, disseminated intravascular coagulation and vasculitis. The complications of splenic Infarct include pseudocyst, haemorrhage, rupture, aneurysm and rarely abscess formation due to infection of the infarcted region. Splenic abscess is a rare and potentially lethal clinical condition. We present a case of a 24-year-old male who was diagnosed with splenic infarct because of scrub typhus and later developed splenic abscess.

Spleen is a lymphatic organ present in the left hypochondrium of the abdomen. It filters and stores the red cells, produces white blood cells and scavenges microorganisms like bacteria. Any condition leading to the interruption of blood flow to the spleen could cause an infarct. The infarction could be classified as partial or complete based on the extent of involvement. The area of the spleen which had loss of blood supply leading to the death of the area is called splenic Infarct. It could also be due to infection, trauma or certain diseases. Rarely splenic infarcts may lead to formation of abscess. The most commonly occurring symptoms of a splenic abscess pain in the abdomen, fever and nausea are not specific to splenic abscess.[1] Hematogenous spread is the most common cause of splenic abscess.[2]

\section{PRESENTATION OF CASE}

A 25-year-old male patient presented with the chief complaints of intermittent fever and pain abdomen of one-week duration. There was no history of loose stools, constipation, vomiting, or burning micturition.

Patient was previously diagnosed as a case of scrub typhus fever (IgM positive for scrub typhus) with splenic infarct 3 and half months back (Fig 1) and treated successfully with doxycycline at that time and was discharged after he had no fever spike for 5 days. The patient was asymptomatic for the next 8 weeks. In between he had 2 follow up visits, last was 3 weeks before this presentation and was asymptomatic.

On examination, the patient was conscious, oriented. Pulse $-112 / \mathrm{min}$, regular. BP $-110 / 70 \mathrm{mmHg}$, right arm supine position. Respiratory rate $-20 / \mathrm{min}$. Per abdomen examination revealed guarding, tenderness in left hypochondrium. Spleen was palpably tender.
Corresponding Author: Dr. Sourya Acharya,

Professor,

Department of Medicine,

Datta Meghe Institute of Medical Sciences, Deemed to be University, Sawangi, Meghe, Wardha, Maharashtra, India.

E-mail: souryaacharya74@gmail.com

DOI: $10.14260 / \mathrm{jemds} / 2020 / 519$

How to Cite This Article:

Annadatha A, Acharya S, Andhale A, et al. Splenic abscess complicating scrub typhus induced splenic infarct- a rare case report. J Evolution Med Dent Sci 2020;9(33):23942396, DOI: 10.14260/jemds/2020/519

Submission 12-04-2020,

Peer Review 06-07-2020,

Acceptance 14-07-2020,

Published 17-08-2020.

Copyright (C) 2020 JEMDS. This is an open access article distributed under Creative Commons Attribution License [Attribution 4.0 International (CC BY 4.0)] 


\section{Investigations}

$\mathrm{Hb}$ - 11.8 gram \%, TLC - 16700 cells/cumm with $88 \%$ neutrophils. Kidney and liver function tests were normal. CECT Abdomen revealed large ill-defined multi lobulated thick walled peripherally enhancing hypodense collection within splenic parenchyma suggesting splenic abscess (Fig 2)

The splenic abscess was drained with a pigtail catheter (Fig 3) and was started on higher antibiotics for the same. The pus was sent for culture and sensitivity which revealed no growth of organism. Pig tail was removed after the drain had less than $25 \mathrm{~mL}$ of aspirate for $48 \mathrm{hrs}$. The patient was put on a prolonged course of antibiotics, and was discharged after the patient was afebrile.

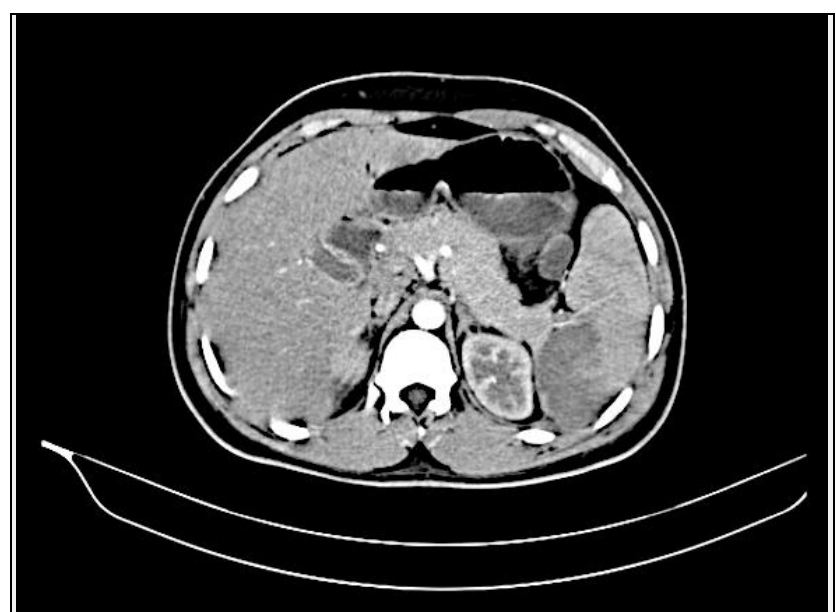

Figure 1. CECT Abdomen Showing Spleen Measuring $12.3 \mathrm{~cm}$, Slightly Enlarged with e/o III Regular Shape Non-Enhancing Hypodense Noted in the Posterior Aspect of Splenic Parenchyma of Approx. Size 9.7 x 5.1 x 3.7 cms Extending from Hilum till Posterior Capsule with Intact Capsule s/o Large Splenic Infarct

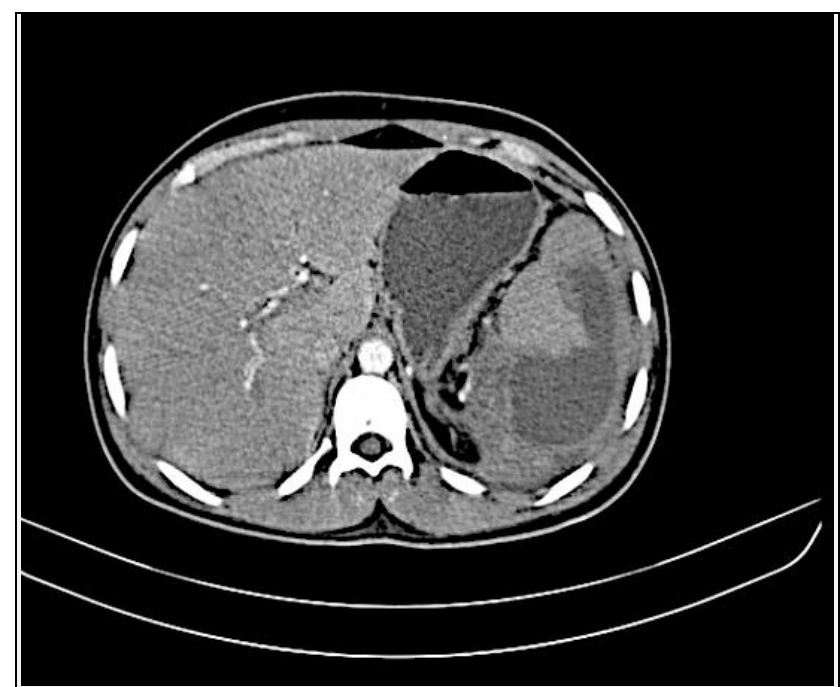

Figure 2. CECT Abdomen Showing Spleen Measuring $13.2 \mathrm{cms}$ with e/o a Large III-Defined Multi-Lobulated Thick Walled Peripherally Enhancing Hypodense Collection within the Splenic Parenchyma with Subcapsular Extension, Enhancing Inner Rim and Hypodense Outer Rim, Measuring Approximately 11 x 7.8 × $7.7 \mathrm{~cm}$ Predominantly in the Posterior Aspect s/o Splenic Abscess

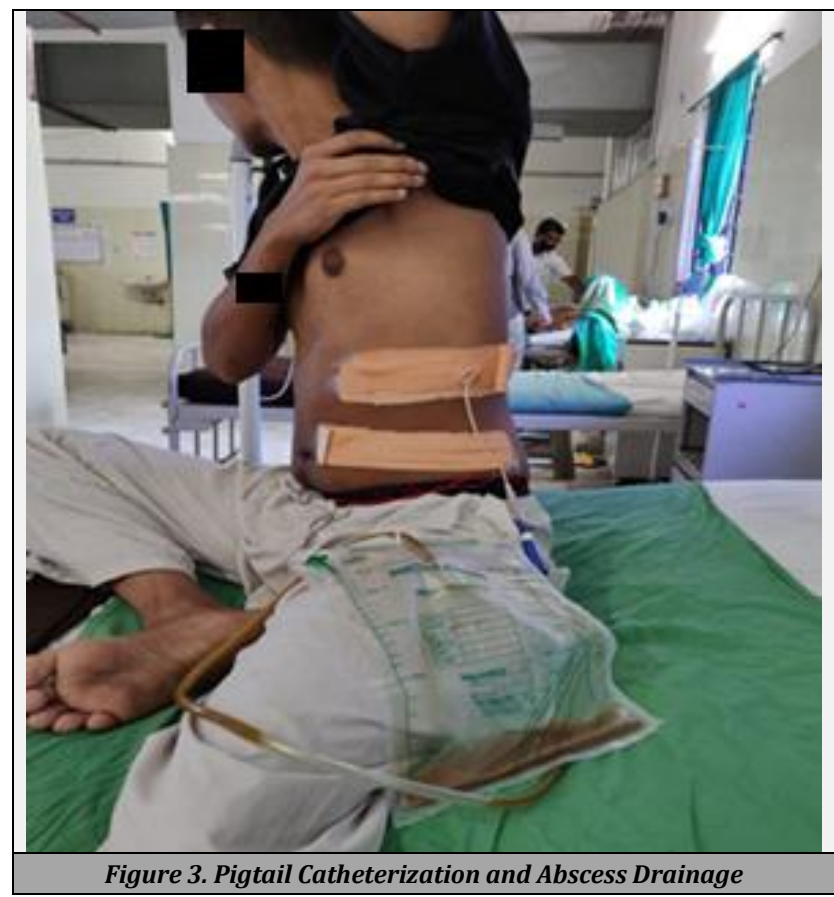

The drained pus was consecutively sent for culture and sensitivity. There was no growth. He was treated with intravenous antibiotics and percutaneous drainage by using pigtail catheterization.

\section{DISCUSSION}

Splenic abscess although not common, should not be to be missed as it carries high risk of mortality. And with timely management, the mortality risk can be greatly avoided to an extent of less than $1 \%$. Although routinely diagnosed on ultrasound, CT is a better investigation modality that also helps in knowing the extent of the abscess and is also helpful in insertion of pig-tail catheterisation. Splenic abscess occurs due to bacteraemia secondary to pooling of blood near the spleen as a result of various conditions. The risk factors for splenic abscess includes splenic infarction, metastasis, neoplasms and diabetes mellitus. ${ }^{[3]}$ In this case, the previously diagnosed splenic infarct was the cause of the abscess.

Although, the gold standard treatment of choice of splenic abscess is splenectomy; studies have shown success using different types of approaches based on characteristics of abscesses. Percutaneous aspiration may be a less invasive option in patients who are at high risk for surgery, or a temporary solution used as a bridge to surgery, avoiding the risk of a fulminant and potentially life-threatening infection. ${ }^{[4]}$ A percutaneous aspiration is a successful approach when the abscess collection is unilocular or bilocular, with a complete and thick wall and no internal septations. Aspiration is easier to achieve when the content is liquid enough to be drained. If there are multiple collections, or there is associated coagulopathy, either laparoscopic or open surgical treatment is preferred. 


\section{Jemds.com}

High-dose parenteral broad-spectrum antibiotics are of paramount importance while further diagnostic and therapeutic arrangements are made. The culture results guide the choice of antibiotics. ${ }^{[5,6,7]}$ There has been previous literature of splenic abscess in sickle cell patients and in Infective endocarditis patients, ${ }^{[8,9]}$ but Scrub typhus vasculitis leading to splenic Infarct and finally leading to splenic abscess has not been reported.

\section{REFERENCES}

[1] Tung CC, Chen FC, Lo CJ. Splenic abscess: an easily overlooked disease? Am Surg 2006;72(4):322-5.

[2] Brook I, Frazier EH. Microbiology of liver and spleen abscesses. J Med Microbiol 1998;47(12):1075-80.

[3] Waheed A, Mathew G, Zemaitis MR. Splenic abscess. In: StatPearls. Treasure Island (FL): StatPearls Publishing 2020.

https://www.ncbi.nlm.nih.gov/books/NBK519546/

\section{Case Report}

[4] van Tienhoven AJ, Lambers WM, Tan TP, et al. Splenic abscess. Ned Tijdschr Geneeskd 2017;161:D1864.

[5] Liverani E, Colecchia A, Mazzella G. Persistent fever and abdominal pain in a young woman with Budd-Chiari syndrome. Gastroenterology 2018;154(3):495-7.

[6] Abou AM, Saint MO, Bercault N, et al. Splenic abscess. A rare pathology requiring a multidisciplinary approach. J Med Liban 2000;48(1):29-33.

[7] Chen H, Hu ZQ, Fang Y, et al. A case report: splenic abscess caused by Burkholderia pseudomallei. Medicine (Baltimore) 2018;97(26):e11208.

[8] Al-Salem AH, Qaisaruddin S, Al Jam'a A, et al. Splenic abscess and sickle cell disease. Am J Hematol 1998;58(2):100-4.

[9] Lindsey ME, Holley CT, Ramaswamy A, et al. Infective endocarditis, embolic stroke and splenic abscess: a case report. J Surg Case Rep 2017;2017(9):rjx147. 